\title{
Aquaporin-4 antibody isoform binding specificities do not explain clinical variations in $\mathrm{NMO}$
}

\section{OPEN}

Joanna Kitley, DPhil Mark Woodhall, PhD M. Isabel Leite, DPhil Jackie Palace, MD Angela Vincent, FRS Patrick Waters, PhD

Correspondence to Prof. Vincent: angela.vincent@ndcn.ox.ac.uk

Supplemental data at Neurology.org/nn

\section{ABSTRACT}

Objective: To assess the clinical relevance of the differential binding of antibodies against the 2 main aquaporin-4 (AQP4) isoforms in neuromyelitis optica (NMO) patient sera using stably transfected human embryonic kidney cells.

Methods: Flow cytometry of human embryonic kidney cells stably transfected with either M23 or M1 AQP4 was used to measure antibody endpoint titers in 52 remission samples and 26 relapse samples from 34 patients with clinically well-characterized AQP4 antibody-positive NMO/NMO spectrum disorder.

Results: The AQP4 M23 $(40-61,440)$ and AQP4 M1 $(<20-20,480)$ titers varied widely between patients, as did the M23:M1 antibody ratio (1-192). In 76 of 78 samples, binding to M23 was higher than binding to $M 1$, including during relapses and remissions ( $<0.0001)$, and the M23:M1 ratio was relatively constant within an individual patient. Titers usually fell after immunosuppression, but the titers at which relapses occurred varied markedly; no threshold level for relapses could be identified, and relapses could occur without a rise in titers. Relapse severity did not correlate with M23 or M1 antibody titers, although there was a correlation between the earliest M23 titers and annualized relapse rates. The M23:M1 ratio and absolute M23 and M1 titers did not relate to age at disease onset, ethnicity, disease severity, phenotype, or relapses at different anatomical sites.

Conclusion: Relative AQP4 antibody binding to M23 and M1 isoforms differs between patients but there is no consistent association between these differences and clinical characteristics of disease. Nevertheless, the M23 isoform provided a slightly more sensitive substrate for AQP4antibody assays, particularly for follow-up studies. Neurol Neuroimmunol Neuroinflamm 2015;2: e121; doi: 10.1212/NXI.0000000000000121

\section{GLOSSARY}

$\mathbf{A C h R}=$ acetylcholine receptor; $\mathbf{A Q P 4}=$ aquaporin-4; $\mathbf{A R R}=$ annualized relapse rate; $\mathbf{C B A}=$ cell-based assay; $\mathbf{D M E M}=$ Dulbecco modified Eagle medium; EDSS = Expanded Disability Status Scale; EDTA = ethylenediaminetetraacetic acid; FACS = fluorescence-activated cell sorting; FCS = fetal calf serum; HEK = human embryonic kidney; $\mathbf{I g}=$ immunoglobulin; LETM = longitudinally extensive transverse myelitis; MGFI = median green fluorescence intensity; NMO = neuromyelitis optica; NMOSD = NMO spectrum disorder; OAPs = orthogonal arrays of particles; $\mathbf{O N}=$ optic neuritis .

Neuromyelitis optica (NMO) is a severe autoimmune inflammatory disorder characterized by optic neuritis $(\mathrm{ON})$ and longitudinally extensive transverse myelitis (LETM). Limited phenotypes, known as NMO spectrum disorders (NMOSDs), are now recognized and include recurrent $\mathrm{ON}$ or, more commonly, monophasic or recurrent LETM. Antibodies to the water channel, aquaporin-4 (AQP4), are found in most patients, act as a disease biomarker, and are thought to be pathogenic. ${ }^{1-4} \mathrm{AQP} 4$ is expressed predominantly on astrocytes in 2 main forms. The AQP4 M23 isoform lacks a 22 amino acid intracellular N-terminus compared with the fulllength AQP4 M1 isoform (hereafter M23 and M1). M23, but not M1, clusters at the cell surface to form orthogonal arrays of particles (OAPs) that appear to enhance antibody binding and complement activation. ${ }^{5,6}$ NMOSD patient sera usually bind more strongly to the M23 isoform, ${ }^{7-9}$ and there is a wide range of relative binding affinities for the 2 isoforms between

From the Nuffield Department of Clinical Neurosciences, John Radcliffe Hospital, University of Oxford, Oxford, UK.

Funding information and disclosures are provided at the end of the article. Go to Neurology.org/nn for full disclosure forms. The Article Processing Charge was paid by the authors.

This is an open access article distributed under the terms of the Creative Commons Attribution-NonCommercial-NoDerivatives License 4.0 (CC BY-NC-ND), which permits downloading and sharing the work provided it is properly cited. The work cannot be changed in any way or used commercially. 
patients. ${ }^{8}$ However, whether differences in the specificity for the 2 isoforms are of clinical significance has not been systematically studied. We measured antibody binding to M1 and M23 isoforms expressed on human embryonic kidney (HEK) 293 cells in sera from 34 patients with clinically well-characterized NMO and NMOSD and related the findings to clinical features.

METHODS Patients and sera. Clinical and serologic studies on patients seen by the UK National NMO specialist service were approved by the regional ethics committee and patients gave written consent.

Sera from 34 patients with NMO/NMOSD were collected prospectively at outpatient visits and during relapses from September 2010 to September 2012 and stored at $-20^{\circ} \mathrm{C}$. Some patients also had sera stored from before September 2010. All patients had been positive for AQP4 antibodies on at least 1 sample in routine clinical cell-based assays (CBAs) using the M23 isoform. ${ }^{10,11}$ Clinical data were collected prospectively at clinic visits and during hospital stays and stored anonymously in a computerized database.

Relapses were defined as the occurrence of new neurologic symptoms and signs and/or new MRI lesions. Relapse serum samples were taken within 14 days of relapse onset, with the exception of onset attack samples that were taken at first presentation to our service, within 3 months of the onset of neurologic symptoms. Remission samples had to be taken at least 28 days after the last relapse and more than 28 days before the next relapse.

Ethics. Oxfordshire REC A (07/Q1604/28 Immune factors in neurological diseases) for the study of any patients whose samples have been referred for testing. Since January 2010, data on all patients seen within the Oxford clinical NMO service have been entered prospectively into a clinical database and patient serum samples routinely tested for AQP4 antibodies and myelin oligodendrocyte glycoprotein antibodies.

Stable M23 and M1 cell lines. Complementary DNA encoding human M1 or M23 AQP4 was subcloned into pIRES-dsRed2 and transfected individually into HEK293A cells overnight using standard polyethylenimine transfection methods. The next day the culture medium (Dulbecco modified Eagle's medium [DMEM] $/ 1 \%$ fetal calf serum [FCS]/penicillin/streptomycin/ amphotericin B) was replaced and supplemented with $200 \mu \mathrm{g} / \mathrm{mL}$ geneticin. After 2-3 weeks, the cells expressing the highest dsRed 2 signal were sorted by flow cytometry (fluorescenceactivated cell sorting [FACS]) and cultured again in selection medium. This procedure was repeated twice to generate stable M1 and M23 cell lines.

Flow cytometry to measure M23 and M1 AQP4 antibody titers. M23 and M1 AQP4 antibody titers in 78 samples (26 relapse samples, 52 remission samples) from the 34 patients with NMO/NMOSD were measured by FACS using the M1 and M23 stable cell lines (hereafter termed M23R and M1R cells). To prevent cell loss, patient sera were first heat-inactivated for 30 minutes at $56^{\circ} \mathrm{C}$ to destroy complement. The individual stable lines were each mixed in a ratio of $3: 1$ with untransfected HEK293 cells, which acted as an inbuilt negative control, and suspended in FACS buffer (DMEM containing $20 \mathrm{mM}$ 4-(2-hydroxyethyl)-1-piperazine ethanesulfonic acid,
$1 \%$ FCS, and $1 \mathrm{mM}$ ethylenediaminetetraacetic acid [EDTA]) at a concentration of $1 \times 10^{6}$ cells $/ \mathrm{mL}$. Cells were rotated at $4^{\circ} \mathrm{C}$ for 2 hours, and $1 \times 10^{5}$ cells were incubated with patient sera (doubling serial dilutions starting at 1:20) for 30 minutes at $4^{\circ} \mathrm{C}$. Cells were washed and bound human immunoglobulin (Ig) G was detected with Alexa Fluor 488 goat anti-human IgG for 1 hour at $4^{\circ} \mathrm{C}$ (Invitrogen, Waltham, MA; 1:500 dilution). Cells were washed again, resuspended in phosphate-buffered saline/2 mM EDTA, and analyzed by FACS.

Cell-based assays to measure M23 and M1 AQP4 antibody titers. Endpoint titers for 41 sera from 18 patients were also determined by visual scoring (0-4) of CBAs using transiently transfected HEK cells, as in routine M23 AQP4 assays. ${ }^{10-12}$ The lowest concentration sample scoring $\geq 1$ was taken as the titer. The mean of 2 experiments was used as the final titer for each sample.

Statistical analysis. Statistical analysis was performed using GraphPad Prism version 4. The Mann-Whitney $U$ test was used to compare 2 groups and the Kruskal-Wallis test was used to compare multiple groups. Correlations were assessed using Spearman correlation coefficient. Statistical significance was set at $p<0.05$.

RESULTS Patient demographics and clinical data. Sera from 30 female patients and 4 male patients aged between 2.8 and 74.9 years (median 37.0 years) were studied. Twenty-five were of Caucasian ethnicity, 7 were of Afro-Caribbean ethnicity, and 2 were of Asian ethnicity.

Median follow-up duration was 75.9 months (range 2.3-403.9 months). Nine patients had a monophasic illness (7 monophasic LETM, 2 monophasic $\mathrm{ON}$ ) and the remainder had a relapsing disease course. Of these, 8 had a typical NMO phenotype with relapses of both ON and LETM, 9 had relapsing LETM, 6 had relapsing $\mathrm{ON}$, and 2 had recurrent brain or brainstem relapses with or without ON or LETM.

The median number of relapses including the onset attack was 4.0 (range 1-10), and the median annualized relapse rate (ARR) for the group (excluding the 1 patient with a follow-up duration $<6$ months) at last follow-up was 0.64 (range 0.092.82). Median Expanded Disability Status Scale (EDSS) score at last follow-up was 5.0 (range 0-8.5).

Relative M23 and M1 antibody titers. For the FACS assays, the stably transfected cells were identified by high red signal (dsRed2) after gating (gate R1, figure 1A; gate R2, figure 1B). Untransfected and poorly expressing stable cells were gated separately to quantify nonspecific binding to HEK cells (gate R3, figure 1B). AQP4 antibody binding was detected in the green channel. A FACS score for each sample was calculated by subtracting the median green fluorescence intensity (MGFI) of the untransfected cells (R3 gate) from the MGFI of the stable AQP4-expressing cells (R2 gate).

Binding curves were plotted for each serum using doubling dilutions; figure $1 \mathrm{C}$ shows an example. 
Figure 1 Cell gating and binding curve from FACS experiments and M23 and M1 antibody titers during relapses and remission

A

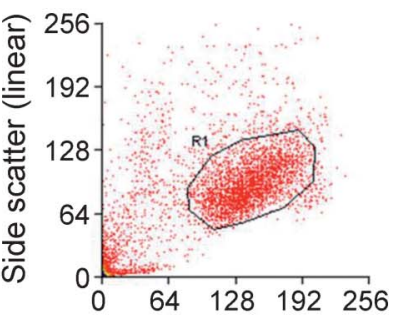

C

Forward scatter (linear)

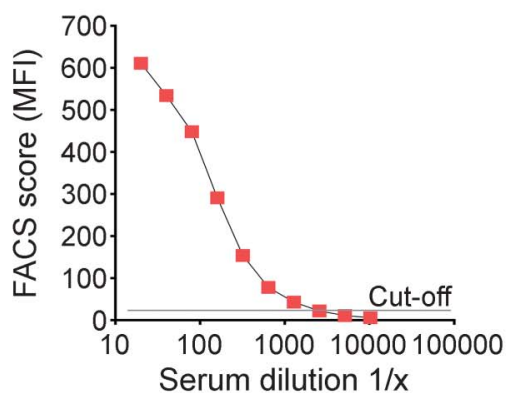

$\mathrm{B}$

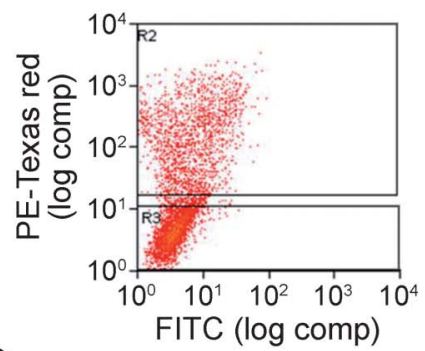

D

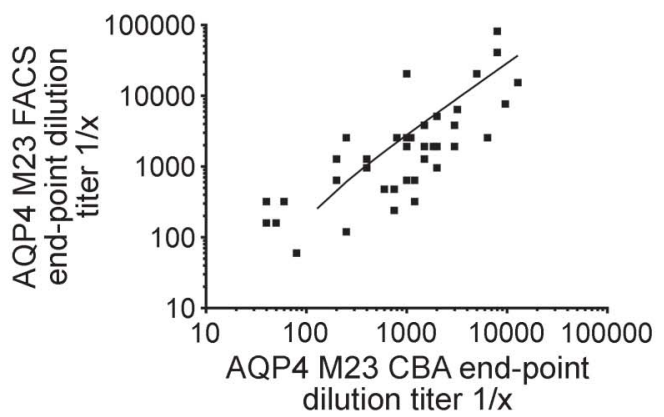

$\mathrm{F}$
E

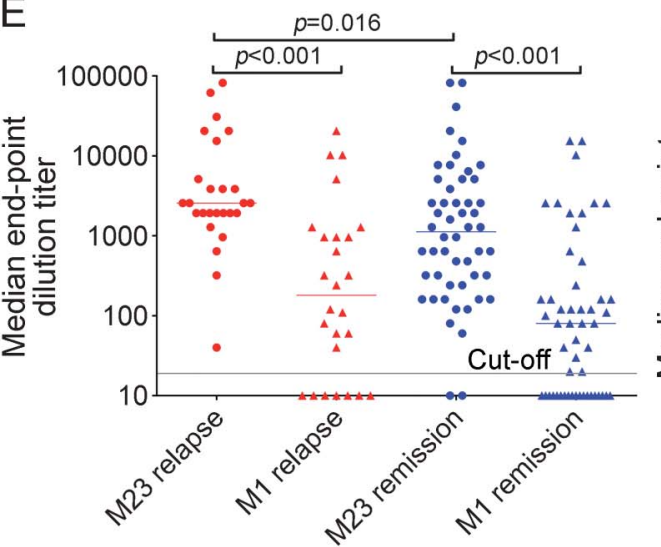

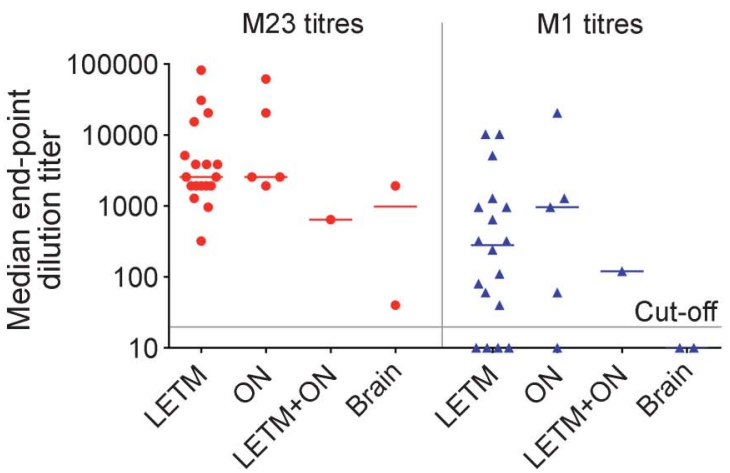

Cell gating for fluorescence-activated cell sorting (FACS) experiments showing (A) gating of human embryonic kidney 293 cells based on cell size and granularity (R1 gate) and (B) gating of M23R cells to differentiate stable dsRed2/aquaporin-4 (AQP4)-expressing cells (R2 gate) from untransfected and poorly expressing cells (R3 gate). (C) Example of a binding curve of serial doubling dilutions of an individual serum. The binding curve was sigmoidal and the endpoint titer was taken as the dilution of the last sample to score above the cutoff. (D) Correlation between FACS and cell-based assay (CBA) endpoint dilution titers. There was a strong correlation between the 2 techniques $(r=0.802 ; p<0.0001)$. (E) Relative levels of M23 and M1 antibodies during relapse and remission. Median M23 antibody titers were higher than M1 antibody titers during both relapse (2,560 vs $180 ; p<0.0001$ ) and remission (1,120 vs $80 ; p<0.0001)$. Median M23 antibody titers were significantly higher during relapse than M23 antibody titers during remission (2,560 vs 1,120; $p=0.0159$ ). Median M1 antibody titers were also higher during relapse than during remission, but this result did not reach significance ( 180 vs $80 ; p=0.1822$ ). (F) Median M23 and M1 antibody titers from all relapse samples were plotted according to anatomical site of attack. KruskalWallis analysis revealed no significant difference in M23 or M1 antibody titers between different attack types $(p=0.1279$; $p=0.2895)$. Note log scale. FITC = fluorescein isothiocyanate; LETM = longitudinally extensive transverse myelitis; MFI = mean fluorescence intensity; $\mathrm{ON}=$ optic neuritis.

The final dilution scoring greater than the cutoff (mean +2 SD of a control cohort comprising 32 healthy and multiple sclerosis control samples; equivalent to an MGFI of 21) was used as the titer for each sample. The mean of 2 experiments was used as the final titer; titers were within a single doubling dilution on repeat assaying for $95 \%$ of all samples run.
To compare the results with those measured using visual scoring of binding to transiently transfected AQP4 M23 cells, as performed for routine diagnosis, endpoint titers were obtained for 41 samples. Although these results were obtained earlier and without knowledge of the FACS results, there was a good correlation between the results of the 2 assays (figure $1 \mathrm{D} ; r=0.802 ; p<0.0001)$. 
All titration values from the FACS assays are shown in figure 1E. M23 antibodies were detectable in all patients in at least 1 sample. M23 antibody titers were higher than M1 antibody titers in 76 of the 78 samples; 2 samples (from 2 different patients) had equal titers. The ratio of M23 to M1 antibody titers varied between patients (range 1-192) and was not predicted by endpoint dilutions of either isoform. The highest M23 antibody titer without detectable M1 antibody was 5,120 and the lowest M23 antibody titer with detectable M1 antibody was 160 .

Comparison between relapse and remission titers. Twenty-six relapse samples were available from $17 \mathrm{pa}$ tients (range 1-6 relapses per patient). Twelve of these 17 patients also had remission samples available. All patients had detectable M23 antibody during relapse, but the titers at which relapses occurred varied markedly (median 2,560; range 40-81,920). Overall, median M23 antibody titers were significantly higher during relapse than during remission (figure $1 \mathrm{E} ; p=$ 0.016). M1 antibody titers at relapse also varied markedly across patients and were negative in some patients (median 180; range $<20-20,480$ ), with a trend toward higher values during relapse than during remission $(p=$ 0.182 ). Median M23 antibody titers were significantly higher than median M1 antibody titers during both relapse $(p<0.0001)$ and remission $(p<0.0001)$.

Paired samples from relapses and subsequent remission periods were available in 11 patients. Neither M23 antibody nor M1 antibody titers were significantly higher during relapse than during remission in these paired samples $(p=0.191$ for M23; $p=$ 0.577 for M1; Wilcoxon matched pairs test).

The 26 relapse samples were taken largely from LETM ( $\mathrm{n}=18)$ or ON ( $\mathrm{n}=5)$ attacks; only 1 sample was taken from an attack of simultaneous LETM/ $\mathrm{ON}$ and 2 samples were taken from attacks affecting the brain and/or brainstem. There were no differences in median M23 antibody $(p=0.128)$ or M1 antibody titers $(p=0.290)$ between the different types of attacks, although M1 antibodies were undetectable in both brain attack samples (figure 1F).

Titers and disease severity. Relapse severity (measured by nadir EDSS score) did not correlate with either M23 or M1 antibody titers $(r=-0.187, p=$ 0.361 for $\mathrm{M} 23 ; r=-0.121, p=0.556$ for $\mathrm{M} 1$, all relapses; $r=0.526, p=0.350$ for M23; $r=$ 0.103, $p=0.950$ for M1, only onset attacks).

There was no correlation between M23 and M1 antibody titers performed on the earliest available sample and EDSS at last follow-up $(r=-0.19$, $p=0.288$ for $\mathrm{M} 23 ; r=-0.23 ; p=0.190$ for M1). There was no significant difference in EDSS at last follow-up between patients with no detectable M1 antibodies and patients with detectable M1 antibodies in all samples (median 6.0 vs $5.0 ; p=$ 0.08 ), and ARR did not differ between patients with no M1 antibodies and patients with M1 antibodies in all samples (median 0.49 vs $0.69 ; p=0.50$ ).

There was a weak correlation between earliest M23 antibody titer and ARR $(r=0.36 ; p=$ $0.044)$ but not earliest M1 titer and ARR $(r=$ $0.21 ; p=0.256)$.

Changes in M1 and M23 titers over time. Onset attack samples were available from 5 patients ( 4 patients with LETM onset attacks and 1 with an ON onset attack), all of whom had detectable antibodies to both isoforms. Onset attack severity (measured by nadir EDSS score) did not correlate with either M23 or M1 antibody titers.

AQP4 antibodies were measured longitudinally in 15 patients, most of whom were not undergoing relapses. Examples of serial antibody determinations are shown in figure 2 and figure e-1 at Neurology.org/nn. Over time, changes in M23 and M1 titers within a patient tended to occur in parallel. Although some relapses were associated with a rise in antibody titers compared to a previous remission sample (figure $2 \mathrm{~A}$ ), definite relapses (MRI-proven, new clinical signs) could occur without a rise in antibody titers (figure $2 \mathrm{~B}$ ), and over time relapses tended to occur at lower antibody titers in patients on treatment (figure $2 \mathrm{~B}$ ). Five patients had serum samples available for more than 1 relapse. In 4 of these 5 patients, M23 and M1 antibody titers were highest in the earliest relapse.

Of the 12 patients with both relapse and remission samples, 5 patients had higher M23 antibody titers during a period of remission than during relapse, and the same was true for M1 antibody titers in 3 patients. On the other hand, patients could have extremely high M23 and M1 antibody titers without experiencing a relapse (up to 81,920 and 15,360 , respectively). Two patients had undetectable M1 antibodies during relapses, and 1 of these had undetectable M1 antibodies in 6 of 6 relapse samples (LETM, ON, and brain), with M23 antibodies of 320-2,560.

Archived serum samples from 1 NMOSD patient with a previous history of typical early-onset myasthenia gravis were available from up to 15 years prior to disease onset. While neither M23 nor M1 antibodies were detectable 124 months prior to NMO onset, M23 antibodies became detectable 90 months, and M1 antibodies 87 months, before the first attack. Titers for both isoforms then increased between this time and development of the onset attack (figure 2C), as has been shown previously. ${ }^{13}$

Within individual patients, antibody titers usually decreased following initiation of treatment (e.g., figure 2D). M1 antibody became undetectable in 4 of the 34 patients after treatment, whereas M23 
A

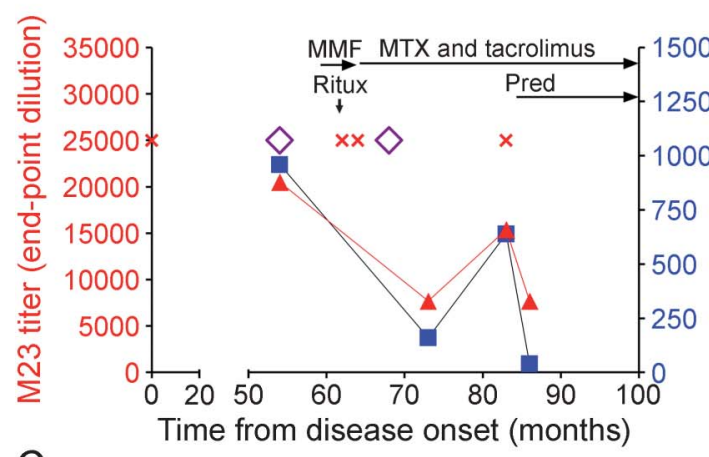

C

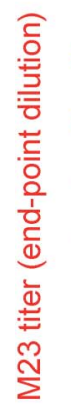

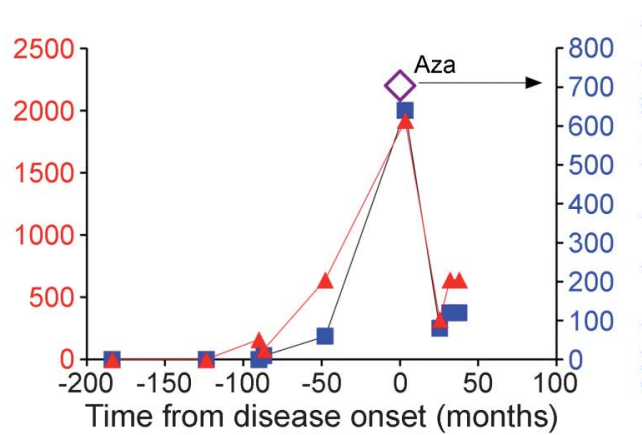

- Rituximab

- Brain relapses $\diamond$ ON relapses

$\times$ TM relapses
$\mathrm{B}$

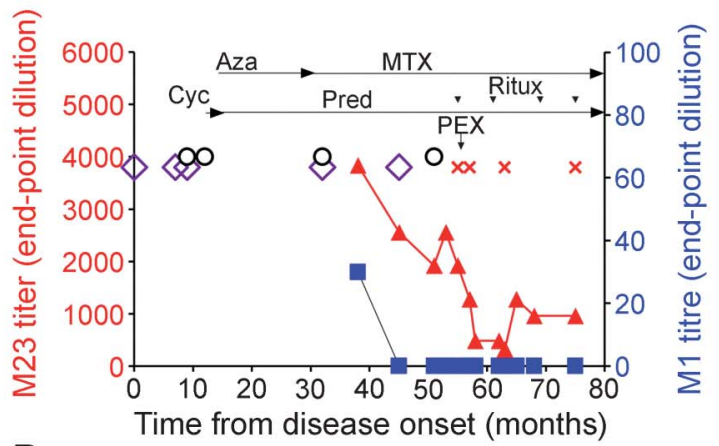

$\mathrm{D}$

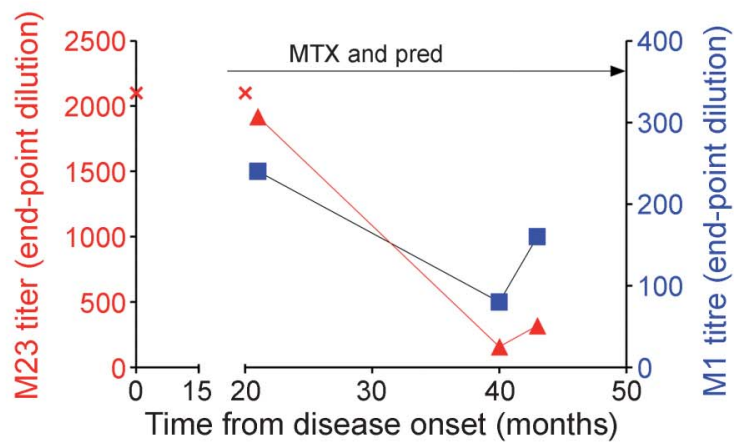

Changes in M23 and M1 antibody titers tended to occur in parallel (A, C, D). Relapses were usually (A), though not always (B), associated with a rise in antibody titers. Archived samples from 1 patient showed that M23 and M1 antibodies were present several years prior to disease onset (C). (D) M23 and M1 antibody titers usually decreased following treatment initiation. Aza = azathioprine; Cyc = cyclosporine; MMF = mycophenolate mofetil; MTX = mitoxantrone; $\mathrm{ON}=$ optic neuritis; PEX = plasma exchange; Pred = prednisone; Ritux = rituximab; $\mathrm{TM}$ = transverse myelitis.

antibodies were still detected at lower levels in all but 1 patient. No significant differences in median M23 or M1 antibody titers between samples taken on $(\mathrm{n}=58)$ or off treatment $(n=20)$ were found, despite the fact that pretreatment samples were often taken during relapses, whereas follow-up samples on treatment were taken during remission. Immunosuppression was stopped in a single patient 89 months after disease onset; this patient has remained relapse-free off treatment for 7.5 years. M23 antibody titers 81 months after treatment cessation are only one-third of what they were at initiation of treatment, and M1 antibody titers are undetectable.

Relationship between M23 and M1 antibody titers and disease phenotype, onset age, and ethnicity. There was no apparent influence of onset age on isoform binding specificity (table). M1 and M23 endpoint dilution titers were measured using the earliest available sample from patients with age at onset $<16$ years $(n=5)$ or age at onset $>70$ years $(n=4)$. There were no significant differences between M23 or M1 antibody titers between the 2 groups (table).

Within the cohort, there were 2 Asian patients, 25 Caucasian patients, and 7 Afro-Caribbean patients. The median M23 and M1 antibody titers using the earliest available sample from these patients did not differ significantly between the 3 ethnic groups (table). Samples from patients with different phenotypes were analyzed. Titers from patients with relapsing ON $(\geq 3$ attacks of ON without involvement of other CNS regions; $\mathrm{n}=6$ ) were compared with samples from patients with relapsing LETM ( $\geq 3$ attacks of LETM without involvement of other CNS regions; $\mathrm{n}=8$ ) and with samples from patients with a full NMO phenotype (attacks of both ON and LETM; $n=8$ ). Only patients with "established" phenotypes were included in this analysis (i.e., patients with only 1 or 2 attacks were excluded unless they had already converted to a full NMO phenotype). M23 and M1 antibody titers were measured using the earliest available sample from each patient, and the median time from disease onset to date of first sample did not differ between the groups $(p=0.148)$. There were no significant differences between median M23 or M1 antibody titers in the 3 phenotype groups (table); this was also the case when only relapse or only remission samples were considered (data not shown).

DISCUSSION There have been several studies demonstrating that sera from patients with NMO react 
Table Influence of patient factors on median M23 and M1 antibody titers

\begin{tabular}{|c|c|c|c|c|}
\hline Demographics and clinical phenotype & Median M23 titer (range) & $p$ Value & Median M1 titer (range) & $p$ Value \\
\hline \multicolumn{5}{|l|}{ Age at disease onset } \\
\hline Young onset $(<16 y ; n=5)$ & $2,560(40-10,240)$ & 0.59 & $160(<20-1,920)$ & 0.99 \\
\hline Late-onset (>70 y; $n=4)$ & $1,760(160-5,120)$ & & $170(<20-5,120)$ & \\
\hline \multicolumn{5}{|l|}{ Ethnicity } \\
\hline Caucasian $(n=25)$ & $2,560(60-20,480)$ & 0.75 & $160(<20-5,120)$ & 0.22 \\
\hline Afro-Caribbean $(n=7)$ & $1,920(40-61,440)$ & & $60(<20-20,480)$ & \\
\hline Asian $(n=2)$ & $1,360(160-2,560)$ & & $160(<20-320)$ & \\
\hline \multicolumn{5}{|l|}{ Disease phenotype } \\
\hline NMO $(n=8)$ & $3,840(240-61,440)$ & 0.36 & $160(<20-20,480)$ & 0.30 \\
\hline rLETM $(n=8)$ & $320(60-15,360)$ & & $95(<20-2,560)$ & \\
\hline $\operatorname{rON}(n=6)$ & $1,280(80-20,480)$ & & $55(<20-1,280)$ & \\
\hline
\end{tabular}

Abbreviations: NMO = neuromyelitis optica; rLETM = relapsing longitudinally extensive transverse myelitis; rON = relapsing optic neuritis.

differently to the $2 \mathrm{AQP} 4$ isoforms, with varied conclusions as to the relevance of these phenomena, but there are no comprehensive studies analyzing patients at different stages of disease in a systematic and quantitative manner. We used FACS analysis on stably transfected cells to determine M23 and M1 antibody titers and their clinical relevance in 34 clinically wellcharacterized patients. Our results confirm previous findings ${ }^{7,8}$ that relative AQP4 antibody binding to M23 and M1 isoforms differs between patients and show conclusively that M23 titers are higher or very rarely equal to M1 titers. However, our results do not provide any evidence that these differences in isoform binding are related to severity or anatomical site of attack, ethnicity, onset age, or disease phenotype.

There was no clear relationship between M23 or M1 binding and patient age or ethnicity, and there were no differences in relative binding specificities between those with typical NMO and those with more limited phenotypes. Moreover, neither M23 nor M1 antibody titers were different between different types of relapses. These findings suggest that it is not isoform binding specificities that dictate anatomical site of attack or the differences in clinical features and disease course in different age and ethnic groups that we reported recently. ${ }^{14}$

Antibodies against both isoforms were present in all available onset attack samples, in contrast to an earlier report demonstrating the appearance of M1 antibody with longer disease duration. ${ }^{7}$ This may reflect differences in cohort selection or M1 assay sensitivity, but M1 antibody tended to disappear over time following initiation of immunosuppression in some patients, and both M1 and M23 titers tended to be lower with successive relapses. Overall, in concordance with other studies, ${ }^{7-9,15}$ antibody binding to the M23 AQP4 isoform was almost always greater than binding to the M1 AQP4 isoform, and while $97 \%$ of all samples were positive for M23 antibody, only $67 \%$ were positive for M1 antibody and no samples were positive for only M1 antibody. Although M1 antibodies were generally undetectable only when M23 antibodies were present at relatively low levels, no threshold cutoff could be identified, and the ratio of M23 to M1 binding varied markedly between patients. Such interpatient variation suggests that the reason for differences in isoform binding is not simply variability in cell surface expression of the 2 isoforms in the assays. One possibility is that AQP4 antibodies bind better to M23 because of divalent binding to clustered M23 in OAPs. An analogous situation in myasthenia gravis has been demonstrated, in which clustering of the acetylcholine receptor (AChR) identified AChR antibodies in patients who were negative for binding to AChR in solution. ${ }^{13}$ Alternatively, as suggested by recent data, it may not be divalent binding due to clustering of M23 that accounts for enhanced reactivity to the M23 isoform ${ }^{8}$; it was proposed that either a neo-epitope or stabilization of a flexible epitope increases affinity when M23 forms higher-order structures such as OAPs. ${ }^{6,8}$ Although these results suggest that M23-AQP4 assays should be used in diagnostic laboratories, many laboratories use M1 DNA that has been reported to produce high coexpression of M23 due to "leaky scanning." ${ }^{16}$ Thus, the sensitivity of these M1 assays may not be as inferior to M23 AQP4 assays as our results might suggest, as found in another recent study comparing the sensitivities between M1 and M23 assays. ${ }^{17}$

There is conflicting evidence as to whether AQP4 antibody titers across patients with NMO are of 
clinical or prognostic significance. ${ }^{10,18-23}$ One problem is that analyses are usually performed across groups, whereas it is apparent that AQP4 antibody titers in relapse and remission vary markedly between individuals. Studies that have looked within individuals have found that relapses are usually associated with a rise in titers but can occur without such a rise. $^{23,24}$ These studies did not provide a definition of relapses, which can be difficult to determine with certainty. Here, using a different methodology and a strict definition of relapses, we have confirmed that relapses can occur without a rise in AQP4 antibody titers. Titers at which relapses occurred varied markedly both between and within individuals and we could not identify a threshold level above which relapses occurred. We also noted that M23 and M1 antibodies were present several years prior to disease onset in 1 patient for whom archived samples were available. Taken together, these findings suggest that the pathogenesis of NMO and the occurrence of relapses must be partially related to factors other than AQP4 antibodies.

Our study has several limitations. First, many subgroup analyses involved small numbers of patients, so we cannot discount the fact that statistical analyses were underpowered to detect any differences. Second, all sera came from patients seen within a specialist NMO referral center, so there may be an element of referral bias. Third, some data were collated retrospectively, which could introduce recall bias with respect to timings of relapses and treatment. However, the majority of patients were inpatients at the time of relapses, so detailed clinical information was available from case notes.

We have shown that the relative binding to the M23 and M1 AQP4 isoforms varies between patients, but we could not identify an association between these differences and any clinical characteristics of disease such as age at onset, clinical phenotype, or ethnicity. Moreover, antibody titers over time did not appear to correlate with disease activity or severity. Binding to M23 was better than binding to $\mathrm{M} 1$, and assays using the M23 isoform should be used in diagnostic laboratories.

\section{AUTHOR CONTRIBUTIONS}

Dr. Kitley was involved in conception and design of the study; collected, analyzed, and interpreted data; and drafted and revised the paper. Dr. Woodhall collected, analyzed, and interpreted data and revised the paper. Dr. Leite collected, analyzed, and interpreted data and revised the paper. Dr. Palace was involved in conception and design of the study, interpreted data, provided supervision, and revised the manuscript. Prof. Vincent was involved in conception and design of the study, interpreted data, provided supervision, drafted and revised the manuscript, and is the guarantor. Dr. Waters developed the stable cell lines; was involved in conception and design of the study; collected, analyzed, and interpreted data; provided supervision; and revised the paper.

\section{STUDY FUNDING}

The study received no specific funding but the authors would like to thank Kevin Clark for help with the FACS sorting, and the NHS National Specialized Commissioning Group for Neuromyelitis Optica for salary support (J.K.) and for support of the NMO research (P.W., M.W., J.P., A.V.) and the NIHR Oxford Biomedical Research Centre for supporting the Neuroimmunology Laboratory (P.W., M.W., A.V.).

\section{DISCLOSURE}

J. Kitley has received speaking honoraria from Novartis and Terumo BCT; has received support for scientific meetings from Teva, Novartis, and Biogen Idec; and was supported by the NHS National Specialised Commissioning Group for Neuromyelitis Optica. M. Woodhall is supported by the NHS National Specialised Commissioning Group for Neuromyelitis Optica. M.I. Leite has received speaker honorarium from Biogen Idec, has received travel grants from Novartis, is supported by the NHS National Specialised Commissioning Group for Neuromyelitis Optica and the NIHR Oxford Biomedical Research Centre, and is on the editorial board for Neuromuscular Disorders. J. Palace is on the scientific advisory boards for Merck Serono, Bayer Schering Pharma, Biogen Idec, Teva Pharmaceutical Industries Ltd, Novartis Pharmaceuticals UK Ltd, Sanofi-Aventis, and Alexion; received travel funding and/or speaker honoraria from Merck Serono, Novartis, Biogen Idec, Bayer Schering, and Medimmune; the ISIS innovation limited has filed a patent application for the use of metabolomics as a method to diagnose and stage disease in MS; has consulted for Ono Pharmaceuticals Ltd, Chugai Pharma Ltd, Biogen Idec, and GlaxoSmithKline; and received research support from Bayer, Schering, Merck Serono, Novartis, Department of Health, MS Society, and UK Guthy Jackson Foundation. A. Vincent received travel funding and speaker honoraria from Baxter International Inc and Biogen Inc; is on the editorial board for Neurology; was an associate editor for Brain; received publishing royalties from Athena Diagnostics, Euroimmun AG, Blackwell Publishing, and Mac Keith Press; has consulted for Athena Diagnostics; and received research support from NIHR. A. Vincent and the Nuffield Department of Clinical Neurosciences hold patents and receive royalties and payments for antibody tests. P. Waters is a named inventor on patents for antibody assays and has received royalties, has received a speaker honorarium from Biogen Idec Japan and Euroimmun AG, is a review editor for Frontiers in Molecular Innate Immunity, and is supported by the NHS National Specialised Commissioning Group for Neuromyelitis Optica and the NIHR Oxford Biomedical Research Centre. Go to Neurology.org/nn for full disclosure forms.

Received January 31, 2015. Accepted in final form April 20, 2015.

\section{REFERENCES}

1. Hinson S, Pittock S, Lucchinetti C, et al. Pathogenic potential of IgG binding to water channel extracellular domain in neuromyelitis optica. Neurology 2007;69: 2221-2231.

2. Vincent T, Saikali P, Cavrol R, et al. Functional consequences of neuromyelitis optica-IgG astrocyte interactions on blood-brain barrier permeability and granulocyte recruitment. J Immunol 2008;181:5730-5737.

3. Saadoun S, Waters P, Bell B, Vincent A, Verkman A, Papadopoulos M. Intra-cerebral injection of neuromyelitis optica immunoglobulin G and human complement produces neuromyelitis optica lesions in mice. Brain 2010; 133:349-361.

4. Ratelade J, Zhang H, Saadoun S, Bennett J, Papadopoulos M, Verkman A. Neuromyelitis optica IgG and natural killer cells produce NMO lesions in mice without myelin loss. Acta Neuropathol 2012;123: 861-872.

5. Hinson S, Romero M, Popescu B, et al. Molecular outcomes of neuromyelitis optica (NMO)-IgG binding to aquaporin- 4 in astrocytes. Proc Natl Acad Sci USA 2012;109:1245-1250. 
6. Phuan P, Ratelade J, Rossi A, Tradrantip L, Verkman A. Complement-dependent cytotoxicity in neuromyelitis optica requires aquaporin- 4 protein assembly in orthogonal arrays. J Biol Chem 2012;287:13829-13839.

7. Mader S, Lutterotti A, Di Pauli F, et al. Patterns of antibody binding to aquaporin- 4 isoforms in neuromyelitis optica. PLoS One 2010;5:e10455.

8. Crane J, Lam C, Rossi A, Gupta T, Bennett J, Verkman A. Binding affinity and specificity of neuromyelitis optica autoantibodies to aquaporin-4 M1/M23 isoforms and orthogonal arrays. J Biol Chem 2011;286:16516-16524.

9. Iorio R, Fryer J, Hinson S, et al. Astrocytic autoantibody of neuromyelitis optica (NMO-IgG) binds to aquaporin-4 extracellular loops, monomers, tetramers and high order arrays. J Autoimmun 2013;40:21-27.

10. Waters P, Jarius $S$, Littleton E, et al. Aquaporin-4 antibodies in neuromyelitis optica and longitudinally extensive transverse myelitis. Arch Neurol 2008;65:913-919.

11. Waters P, McKeon A, Leite MI, et al. Serological diagnosis of NMO: a multicentre comparison of aquaporin-4-IgG assays. Neurology 2012;78:665-671.

12. Leite MI, Coutinho E, Lana-Peixoto M, et al. Myasthenia gravis and neuromyelitis optica spectrum disorder: a multicenter study of 16 patients. Neurology 2012;78: 1601-1607.

13. Leite MI, Jacob S, Viegas S, et al. IgG1 antibodies to acetylcholine receptors in "seronegative" myasthenia gravis. Brain 2008;131:1940-1952.

14. Kitley J, Leite MI, Nakashima I, et al. Prognostic factors and disease course in aquaporin- 4 antibody-positive patients with neuromyelitis optica spectrum disorder from the United Kingdom and Japan. Brain 2012;135:1834-1849.

15. Nicchia G, Mastrototaro M, Rossi A, et al. Aquaporin-4 orthogonal arrays of particles are the target for neuromyelitis optica autoantibodies. Glia 2009;57:1363-1373.
16. Pisani F, Sparaneo A, Tortorella C, et al. Aquaporin-4 autoantibodies in Neuromyelitis Optica: AQP4 isoformdependent sensitivity and specificity. PLoS One 2013;8: e79185.

17. Jarius S, Friedmann P, Fechier K, et al. Aquaporin- 4 antibody testing: direct comparison of M1-AQP4-DNAtransfected cells with leaky scanning versus M23AQP4-DNA-transfected cells as antigenic substrate. J Neuroinflammation 2014;11:129.

18. Takahashi T, Fujihara K, Nakashima I, et al. Anti-aquaporin- 4 antibody is involved in the pathogenesis of NMO: a study on antibody titer. Brain 2007;130:1235-1243.

19. Jarius S, Aboul-Enein F, Waters P, et al. Antibody to aquaporin- 4 in the long term course of neuromyelitis optica. Brain 2008;131:3072-3080.

20. Jarius S, Franciotta D, Paul F, et al. Cerebrospinal fluid antibodies to aquaporin- 4 in neuromyelitis optica and related disorders: frequency, origin, and diagnostic relevance. J Neuroinflammation 2010;7:52.

21. Hayakawa S, Mori M, Okuta A, et al. Neuromyelitis optica and anti-aquaporin- 4 antibodies measured by an enzyme-linked immunosorbent assay. J Neuroimmunol 2008;196:181-187.

22. Hinson S, McKeon A, Fryer J, Apiwattanakul M, Lennon V, Pittock S. Prediction of neuromyelitis optica attack severity by quantitation of complement-mediated injury to aquaporin-4-expressing cells. Arch Neurol 2009;66:1164-1167.

23. Isobe $\mathrm{N}$, Yonekawa $\mathrm{T}$, Matsushita $\mathrm{T}$, et al. Clinical relevance of serum aquaporin- 4 antibody levels in neuromyelitis optica. Neurochem Res 2013;38:997-1001.

24. Kim S, Kim W, Li X, et al. Repeated treatment with rituximab based on the assessment of peripheral circulating memory B cells in patients with relapsing neuromyelitis optica over 2 years. Arch Neurol 2011;68:1412-1420. 


\section{Neurology \\ Neuroimmunology \& Neuroinflammation}

Aquaporin-4 antibody isoform binding specificities do not explain clinical variations in NMO

Joanna Kitley, Mark Woodhall, M. Isabel Leite, et al.

Neurol Neuroimmunol Neuroinflamm 2015;2;

DOI 10.1212/NXI.0000000000000121

This information is current as of June 18, 2015

Neurol Neuroimmunol Neuroinflamm is an official journal of the American Academy of Neurology.

Published since April 2014, it is an open-access, online-only, continuous publication journal. Copyright $\odot$ 2015 American Academy of Neurology. All rights reserved. Online ISSN: 2332-7812.

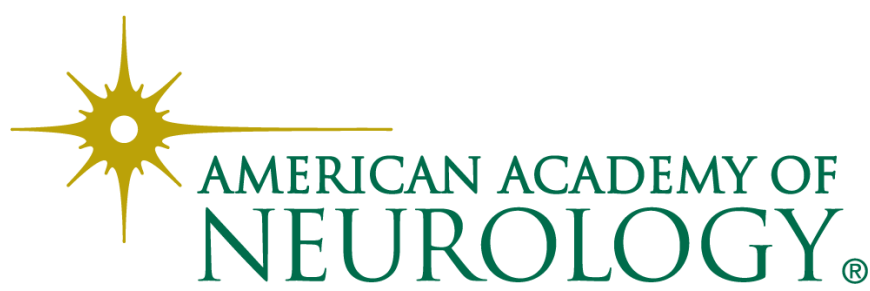




\section{Updated Information \&} Services

\section{Supplementary Material}

\section{References}

Citations

Subspecialty Collections

Permissions \& Licensing

Reprints including high resolution figures, can be found at: http://nn.neurology.org/content/2/4/e121.full.html

Supplementary material can be found at: http://nn.neurology.org/content/suppl/2015/06/18/2.4.e121.DC1

This article cites 24 articles, 4 of which you can access for free at: http://nn.neurology.org/content/2/4/e121.full.html\#\#ref-list-1

This article has been cited by 2 HighWire-hosted articles: http://nn.neurology.org/content/2/4/e121.full.html\#\#otherarticles

This article, along with others on similar topics, appears in the following collection(s):

All Demyelinating disease (CNS)

http://nn.neurology.org//cgi/collection/all_demyelinating_disease_cns Autoimmune diseases

http://nn.neurology.org//cgi/collection/autoimmune_diseases Devic's syndrome

http://nn.neurology.org//cgi/collection/devics_syndrome

Information about reproducing this article in parts (figures,tables) or in its entirety can be found online at:

http://nn.neurology.org/misc/about.xhtml\#permissions

Information about ordering reprints can be found online: http://nn.neurology.org/misc/addir.xhtml\#reprintsus

Neurol Neuroimmunol Neuroinflamm is an official journal of the American Academy of Neurology.

Published since April 2014, it is an open-access, online-only, continuous publication journal. Copyright $\odot$ 2015 American Academy of Neurology. All rights reserved. Online ISSN: 2332-7812.

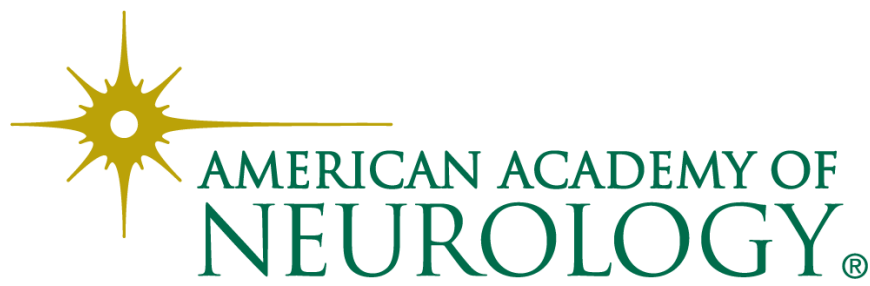

